\title{
Aquarius: The Instrument and Initial Results
}

\author{
D.M. Le Vine \\ NASA/Goddard Space Flight Center, Greenbelt, MD 20771 \\ G.S.E. Lagerloef \\ Earth and Space Research, Seattle, WA 98121 \\ C. Ruf \\ University of Michigan, Ann Arbor, MI 48109 \\ F. Wentz \\ Remote Sensing Systems, Santa Rosa, CA 91109 \\ S. Yueh \\ Jet Propulsion Laboratory, Pasadena, CA 91109 \\ J. Piepmeier \\ NASA/Goddard Space Flight Center, Greenbelt, MD 20771 \\ E. Lindstrom \\ NASA HQ, Washington, D.C. 20546 \\ E. Dinnat \\ Chapman University, Orange, CA 92866
}

\begin{abstract}
Aquarius was launched on June 10, 2011 aboard the Aquarius/SAC-D observatory and the instrument has been operating continuously since the initial turned-on was completed on August 25. The initial observed antenna temperatures were close to predicted and the first salinity map was released in September. In order to map the ocean salinity field, Aquarius includes several special features such as the inclusion of a scatterometer to provide a roughness correction, measurement of the third Stokes parameter to correct for Faraday rotation, and fast sampling to mitigate the effects of RFI. This paper provides an overview of the instrument and an example of initial results. Details are covered in subsequent papers in the session on Aquarius.
\end{abstract}

Keywords-Microwave Remote Sensing; Microwave radiometry; ocean salinity

\section{INTRODUCTION}

Aquarius is the primary instrument on the Aquarius/SAC-D mission, a partnership between NASA and the Argentine space agency (CONAE). Figure 1 shows the observatory in its deployed configuration as it would look on orbit, and Figure 2

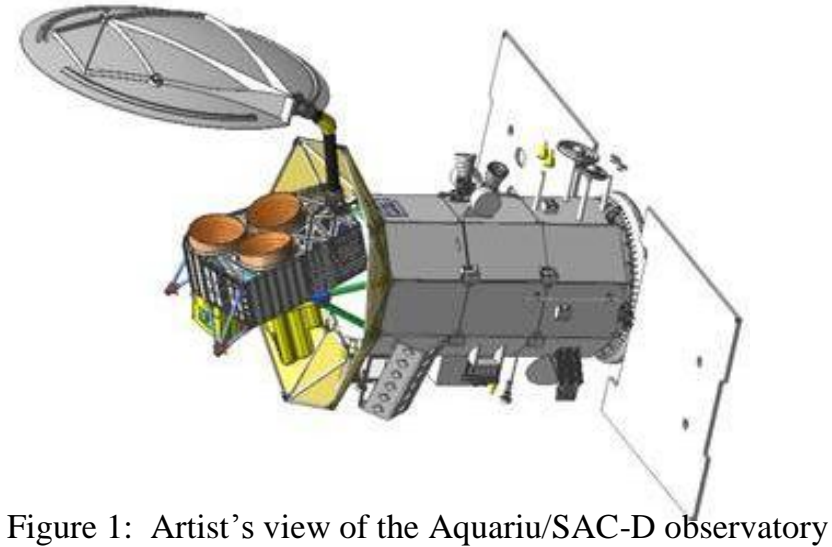

shows the actual hardware in the anechoic chamber while undergoing EMI/EMC testing. (The struts holding the Aquarius reflector in Figure 2 are to offset the load of gravity. In orbit the reflector is supported by the single boom as shown in Figure 1.) Additional instruments, developed by

CONAE and its partners and mounted on the SAC-D bus, include [1]:

- Microwave Radiometer (MWR) with frequencies of 23.8 and $37 \mathrm{GHz}$ to monitor precipitation, wind speed and sea ice developed by CONAE;

- Visible wavelength camera (HSC) developed by CONAE and an infrared camera developed by CONAE and the Canadian Space Agency, CSA;

- A radio occultation sounder (ROSA) provided by the Italian space agency, ASI;

- A space radiation environment experiment provided by the French space agency, CNES.

\section{AQUARIUS}

Aquarius consists of three radiometers whose antenna beams are oriented to look across-track and image in "pushbroom" mode. The observatory as shown in Figure 1 flies out of the page (toward the reader) with its long axis perpendicular to the velocity vector and with the earth surface toward the bottom of the page. The three structures on the bottom (earth-viewing) side of the SAC-D bus are, from left to right, the ROSA antenna, the cameras and the MWR. The actual flight hardware can be seen on the left side of Figure 2.

The three Aquarius radiometer beams image across track as illustrated in Figure 3 which shows the ground track (solid line) and the three beams in color. Two of the beams, the inner and outer beam, are slightly aft of cross track and the 


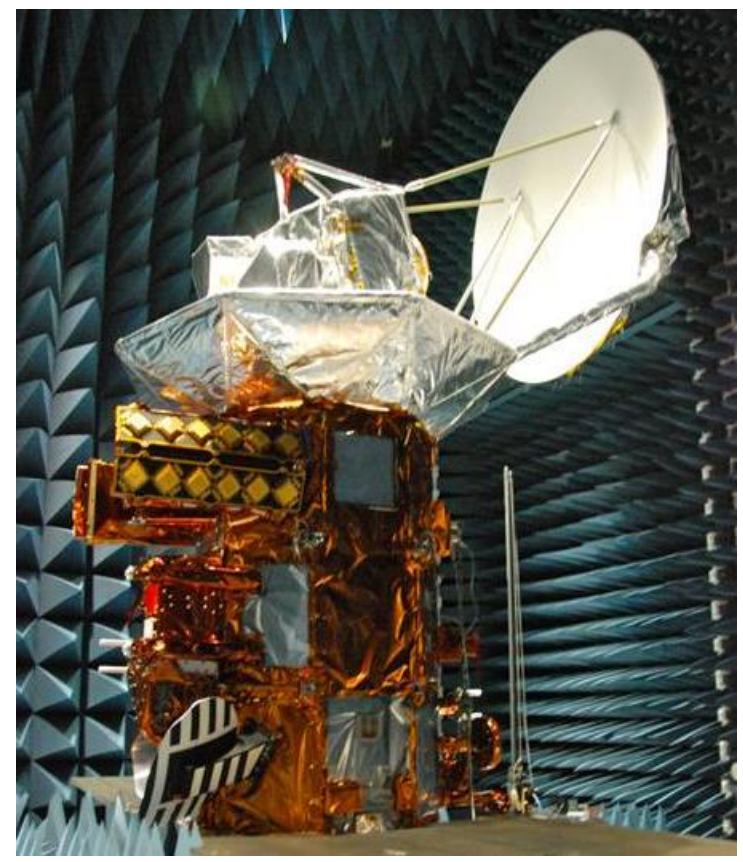

Figure 2: Aquarius in the deployed configuration during EMI/EMC testing

middle beam is slightly forward of cross track. The three beams map a swath of $390 \mathrm{~km}$ which permits mapping of the globe in 7 days. There are three separate radiometers, one for each feed, but only one scatterometer. The scatterometer is designed to have the same $3 \mathrm{db}$ footprint as the radiometer and cycles between the three feeds. The cycling is done rapidly so that the radiometer and scatterometer make measurements at essentially the same time.

The science objective of Aquarius is to monitor the seasonal and inter-annual variation of the large scale features of the surface salinity field in the open ocean. The goal is to provide global maps on a monthly basis with a spatial resolution of $150 \mathrm{~km}$ and an accuracy of $0.2 \mathrm{psu}$. These requirements are derived from the need to better understand the buoyancy driven thermohaline circulation of the ocean and its relationship to climate and the global water cycle [2]. Mapping sea surface salinity is a challenging measurement and to do this Aquarius has several unique features. Among them are:

- The inclusion of a scatterometer viewing the same spot as the radiometer to provide a correction for roughness;

- A polarimetric radiometer channel (third Stokes parameter) to help correct for Faraday rotation;

- Fast sampling to detect and mitigate the effects of RFI;

- Internal calibration (noise sources) and good thermal control.

In addition, special care has been taken to avoid contamination by radiation from the sun which is a significant

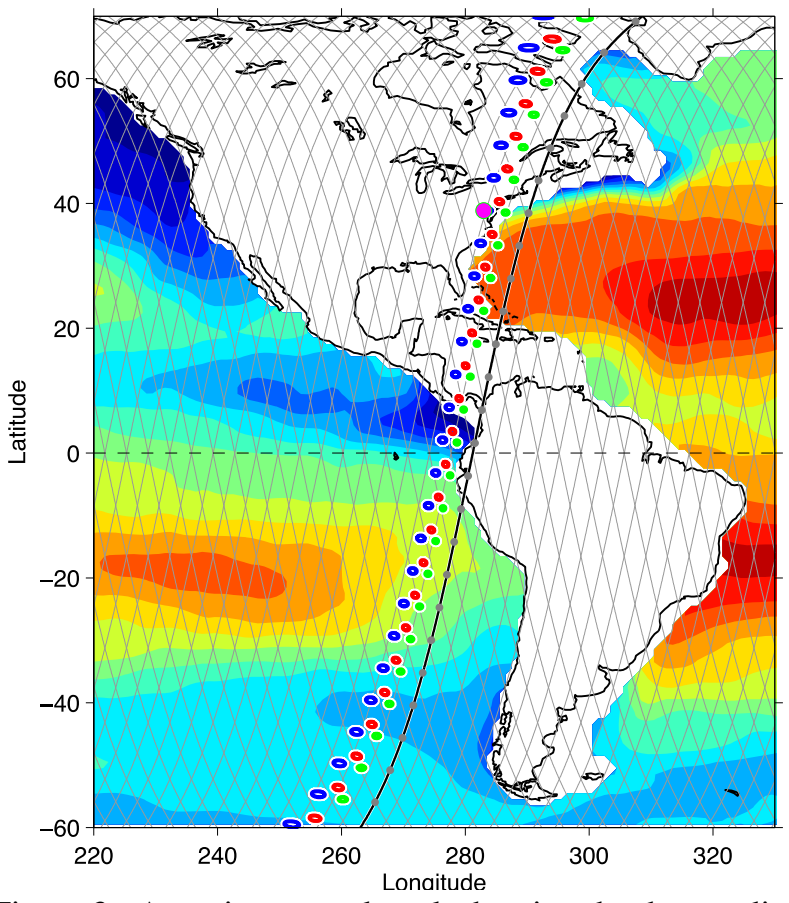

Figure 3: Aquarius ground track showing the three radiometer beams imaging across track.

source of radiation at L-band [3]. In order to avoid solar reflection from the rough ocean into the main beam of the radiometers (i.e. sun "glint"), the observatory flies in a sunsynchronous orbit close to the day-night terminator with the radiometer beams pointed toward the night time side. In addition, during design of the antenna reflector requirements were placed on the magnitude of the side lobes in the direction of the direct ray from the Sun and the ray reflected from the ocean surface toward the spacecraft.

Thermal control is another important feature of Aquarius. The large umbrella-like structure at the interface between Aquarius and the SAC-D observatory "bus" is a sun-shield designed to limit direct solar heating of the radiometer front ends which are mounted on the OMT-feed structure (Figure 5 in [1]). Active thermal control (heaters, reflector and control circuitry) is employed to keep the radiometer front-end temperature stable. The design goal was a maximum variation of less than $0.1 \mathrm{C}$ per orbit. The system is doing better than this in orbit.

\section{Examples}

\section{A. Radio Frequency Interference (RFI)}

It has been known since the early aircraft experiments at Lband that even though the radiometers operate in a protected band (passive use only), RFI is a problem. To help mitigate this problem, Aquarius implemented rapid sampling, with the idea that when RFI was detected only the minimum amount of data would be lost. Measurements are made in $10 \mathrm{~ms}$ blocks and checked for RFI using a "glitch" detector implemented in software [4]. Those data exceeding the threshold are removed and the remainder averaged to form $1.44 \mathrm{sec}$ blocks. These 
are the basic data blocks for processing (i.e. producing calibrated brightness temperature and estimates of SSS). Figure 4 illustrates the global distribution of RFI as detected by Aquarius. The map shows the largest signal detected (peak hold) plotted on the nominal background for one radiometer channel (outer beam and H-pol). The white indicates no data (present because the coverage is incomplete using only one radiometer beam). The pattern is similar to that reported by SMOS and includes expected features such as the DEW line radars in North America. The oceans experience less frequent and less severe RFI than over land, and the current work is focused on reducing false alarms over the oceans and tuning the algorithm to better accommodate differences in ocean and land scenes.

\section{B. Sea Surface Salinity}

The Aquarius radiometers performed remarkably close to predictions based on the forward model assembled to predict the expected antenna temperatures [5]. The forward model was used to adjust biases in the antenna temperatures and in September, 2011 about one month after the instrument was turned on, the first Aquarius map of sea surface salinity was released (Figure 5). It conforms well to climatology and shows detail in areas such as the ITCZ and freshwater outflow from the Amazon River that promise exciting science in the near future. Work continues to improve the product. A small drift in calibration (about $.1 \mathrm{~K}$ per week) has been detected and is being corrected using a model based on the suspected cause. A small difference between ascending and descending orbits has also been detected and its cause is currently under investigation.

\section{REFERENCES}

[1] D. M. Le Vine, G. S. E. Lagerloef, F. R. Colomb, S. H. Yueh, and F. A. Pellerano, "Aquarius: An instrument to monitor sea surface salinity from space, "pace," IEEE Trans. Geosci. Remote Sens., vol. 45, no. 7, pp. 2040-2050, Jul. 2007.

[2] G. Lagerloef et al. "The Aquarius/SAC-D Mission: Designed to Meet the Salinity Remote-Sensing Challenge", Oceanography, Vol 21 (\#1), pp 68-81, March, 2008.

[3] D. M. Le Vine, S. Abraham, F. Wentz, and G. S. E. Lagerloef, "Impact of the Sun on remote sensing of sea surface salinity from space," in Proc.IGARSS, Seoul, Korea, Jul. 2005, vol. 1, pp. 288-291. DOI: 10.1109/IGARSS.2005.1526164.

[4] S. Misra and C. S. Ruf, "Detection of Radio-Frequency Interference for the Aquarius Radiometer, IEEE Trans. Geosci Remote Sens., Vol 46 (\#10), pp 3123-3128, October, 2008

[5] D. M. Le Vine, E.P. Dinnat, S. Abraham, P. de Matthaeis and F.J. Wentz, "The Aquarius Simulator and Cold Sky Calibration", IEEE TGARS, Vol 49 (\#9), pp 3198-3210, September, 2011.

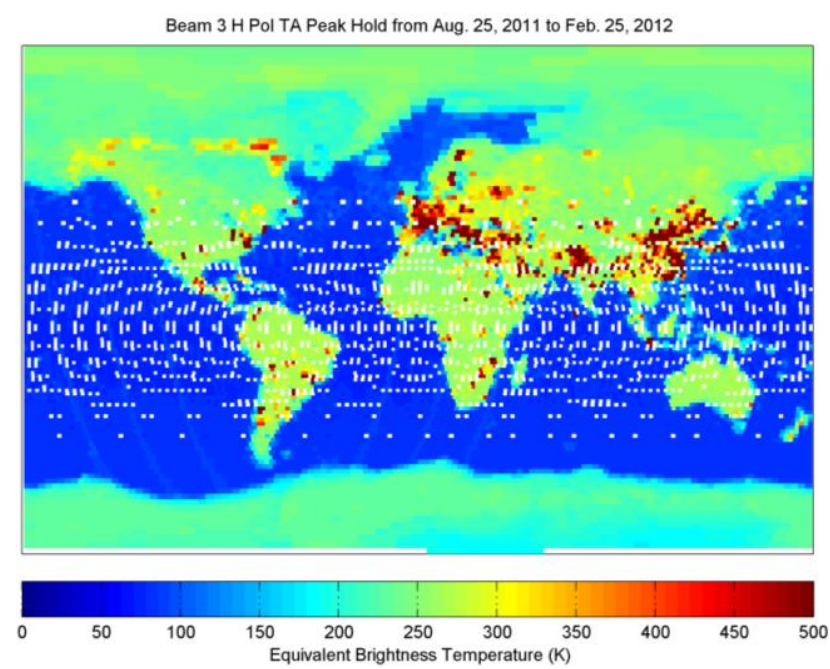

Figure 4: Peak RFI at horizontal polarization in the outer Aquarius beam. White indicates absence of data.

$\mathrm{J}$

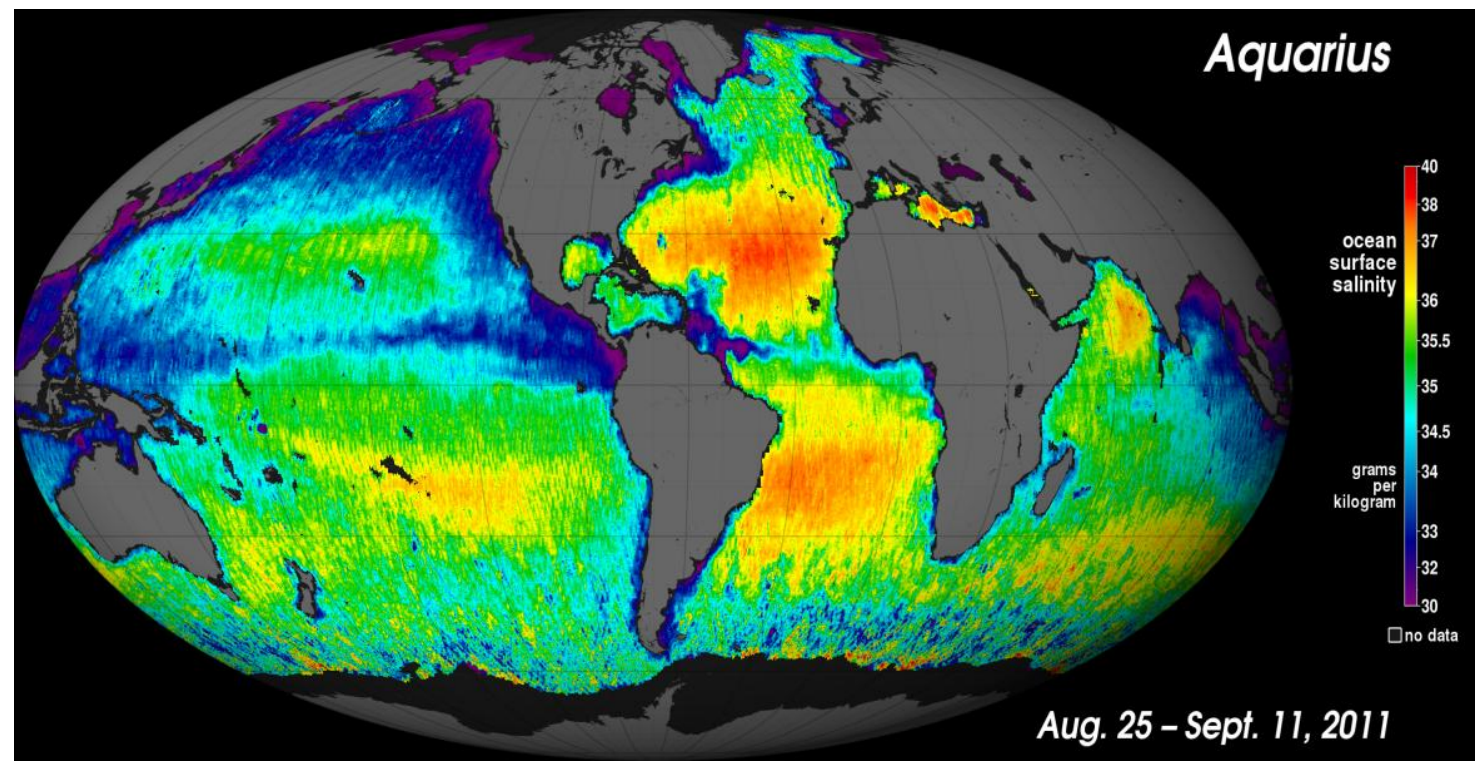

Figure 5: Aquarius first map of the global sea surface salinity field. 